Article

\title{
Universality in Short Intervals of the Riemann Zeta-Function Twisted by Non-Trivial Zeros
}

\author{
Antanas Laurinčikas ${ }^{1,+(1)}$ and Darius Šiaučiūnas ${ }^{2, *,+}$ (I) \\ 1 Institute of Mathematics, Faculty of Mathematics and Informatics, Vilnius University, Naugarduko str. 24, \\ LT-03225 Vilnius, Lithuania; antanas.laurincikas@mif.vu.lt \\ 2 Regional Development Institute, Šiauliai University, P. Višinskio str. 25, LT-76351 Šiauliai, Lithuania \\ * Correspondence: darius.siauciunas@su.lt \\ + These authors contributed equally to this work.
}

Received: 3 October 2020; Accepted: 20 October 2020; Published: 3 November 2020

check for updates

\begin{abstract}
Let $0<\gamma_{1}<\gamma_{2}<\cdots \leqslant \gamma_{k} \leqslant \cdots$ be the sequence of imaginary parts of non-trivial zeros of the Riemann zeta-function $\zeta(s)$. Using a certain estimate on the pair correlation of the sequence $\left\{\gamma_{k}\right\}$ in the intervals $[N, N+M]$ with $N^{1 / 2+\varepsilon} \leqslant M \leqslant N$, we prove that the set of shifts $\zeta\left(s+i h \gamma_{k}\right), h>0$, approximating any non-vanishing analytic function defined in the strip $\{s \in \mathbb{C}: 1 / 2<\operatorname{Res}<1\}$ with accuracy $\varepsilon>0$ has a positive lower density in $[N, N+M]$ as $N \rightarrow \infty$. Moreover, this set has a positive density for all but at most countably $\varepsilon>0$. The above approximation property remains valid for certain compositions $F(\zeta(s))$.
\end{abstract}

Keywords: Montgomery pair correlation conjecture; non-trivial zeros; Riemann zeta-function; universality

MSC: 11M06; 11M26

\section{Introduction}

The Riemann zeta-function $\zeta(s), s=\sigma+i t$, is defined, for $\sigma>1$, by

$$
\zeta(s)=\sum_{m=1}^{\infty} \frac{1}{m^{s}}=\prod_{p}\left(1-\frac{1}{p^{s}}\right)^{-1},
$$

where the infinite product is taken over all prime numbers, and has analytic continuation over the whole complex plane, except for the point $s=1$ which is a simple pole with residue 1 . The function $\zeta(s)$ and its value distribution play an important role not only in analytic number theory but in mathematics in general.

It is well known by a Bohr and Courant work [1] that the set of values of $\zeta(\sigma+i t)$ with any fixed $\sigma \in(1 / 2,1]$ is dense in $\mathbb{C}$. Voronin obtained [2] the infinite-dimensional version of the Bohr-Courant theorem, proving the so-called universality of $\zeta(s)$. This means that every non-vanishing analytic function in the strip $D=\{s \in \mathbb{C}: 1 / 2<\sigma<1\}$ can be approximated by shifts $\zeta(s+i \tau)$. We recall the modern version of the Voronin theorem. Denote by $\mathcal{K}$ the class of compact subsets of the strip $D$ with connected complements, and by $H_{0}(K)$ with $K \in \mathcal{K}$ the class of continuous non-vanishing functions on $K$ that are analytic in the interior of $K$. Then, for $K \in \mathcal{K}, f(s) \in H_{0}(K)$ and every $\varepsilon>0$, the inequality

$$
\liminf _{T \rightarrow \infty} \frac{1}{T} \text { meas }\left\{\tau \in[0, T]: \sup _{s \in K}|\zeta(s+i \tau)-f(s)|<\varepsilon\right\}>0
$$

is true; see, for example, [3-6]. Thus, we have that there are infinitely many shifts $\zeta(s+i \tau)$ approximating a given function $f(s) \in H_{0}(K)$. 
The above theorem is of continuous type because $\tau$ in shifts $\zeta(s+i \tau)$ can take arbitrary real values. If $\tau$ runs over a certain discrete set, then we have the discrete universality that was proposed in [7]. Denote by \#A the cardinality of a set $A$, and suppose that $N$ runs over the set of non-negative integers. If $K$ and $f(s)$ are as above, then we have, for $h>0$ and $\varepsilon>0$,

$$
\liminf _{N \rightarrow \infty} \frac{1}{N+1} \#\left\{0 \leqslant k \leqslant N: \sup _{s \in K}|\zeta(s+i k h)-f(s)|<\varepsilon\right\}>0 .
$$

Approximations of analytic functions by more general discrete shifts were considered in [8-10].

Denote by $\gamma_{1}<\gamma_{2}<\cdots \leqslant \gamma_{k} \leqslant \cdots$ the positive imaginary parts of non-trivial zeros $\rho_{k}=\beta_{k}+i \gamma_{k}$ of the function $\zeta(s)$. Discrete universality theorems with shifts $\zeta\left(s+i h \gamma_{k}\right)$ were obtained in [11,12]. In [11], for this the Riemann hypothesis was used, while in [12], the weak form of the Montgomery pair correlation conjecture [13] was involved. More precisely, the estimate, for $c>0$,

$$
\sum_{\substack{0<\gamma_{k}, \gamma_{l} \leqslant T \\\left|\gamma_{k}-\gamma_{l}\right|<c / \log T}} 1 \ll_{c} T \log T, \quad T \rightarrow \infty,
$$

was required. Analogical results for more general functions were given in $[14,15]$.

On the other hand, all above theorems are non-effective in the sense that any concrete shift approximating a given analytic function is not known. This shortcoming leads to the idea of universality in intervals as short as possible containing $\tau$ with approximating property. The first result in this direction was obtained in [16].

Theorem 1. Suppose that $T^{1 / 3}(\log T)^{26 / 15} \leqslant H \leqslant T, K \in \mathcal{K}$ and $f(s) \in H_{0}(K)$. Then, for every $\varepsilon>0$,

$$
\liminf _{T \rightarrow \infty} \frac{1}{H} \text { meas }\left\{\tau \in[T, T+H]: \sup _{s \in K}|\zeta(s+i \tau)-f(s)|<\varepsilon\right\}>0 .
$$

The aim of this paper is the universality of the function $\zeta(s)$ in short intervals with shifts $\zeta\left(s+i h \gamma_{k}\right)$. In this case, the estimate (1) is not sufficient. Therefore, for $N^{1 / 2+\varepsilon} \leqslant M \leqslant N$ with $\varepsilon>0$, we use the following hypothesis:

$$
\sum_{\substack{k=N \\\left|\gamma_{k}-\gamma_{l}\right|<c / \log N}}^{N+M} \sum_{\substack{l=N \\ N+M}}^{N} 1 \ll_{c} M
$$

which, as estimate (1), also gives a certain information on the pair correlation of non-trivial zeros, differently from estimate (1), however, in short intervals.

Theorem 2. Suppose that $N^{1 / 2+\varepsilon} \leqslant M \leqslant N$, and estimate (2) are true. Let $K \in \mathcal{K}$ and $f(s) \in H_{0}(K)$. Then, for every $\varepsilon>0$ and $h>0$,

$$
\liminf _{N \rightarrow \infty} \frac{1}{M+1} \#\left\{N \leqslant k \leqslant N+M: \sup _{s \in K}\left|\zeta\left(s+i h \gamma_{k}\right)-f(s)\right|<\varepsilon\right\}>0
$$

Moreover, "lim inf" can be replaced by "lim" for all but at most countably many $\varepsilon>0$.

Theorem 2 has a generalization for certain compositions $F(\zeta(s))$. Denote by $H(D)$ the space of analytic functions on the strip $D$ endowed with the topology of uniform convergence on compacta. Moreover, let

$$
S=\{g \in H(D): \text { either } g(s) \neq 0 \text { for all } s \in D \text {, or } g(s) \equiv 0\}
$$


and, for the operator $F: H(D) \rightarrow H(D)$ and distinct complex numbers $a_{1}, \ldots, a_{r}$,

$$
H_{a_{1}, \ldots, a_{r} ; F}(D)=\left\{g \in H(D): g(s) \neq a_{j} \text { for all } s \in D, j=1, \ldots, r\right\} \cup\{F(0)\} .
$$

Then we have

Theorem 3. Suppose that estimate (2) is true, $N^{1 / 2+\varepsilon} \leqslant M \leqslant N$, and $F: H(D) \rightarrow H(D)$ is a continuous operator such that $F(S) \supset H_{a_{1}, \ldots, a_{r} ; F}(D)$. For $r=1$, let $K \in \mathcal{K}$ and $f(s)$ be a continuous $\neq a_{1}$ function on $K$, and analytic in the interior of $K$. For $r \geqslant 2$, let $K$ be an arbitrary compact subset of $D$, and $f(s) \in H_{a_{1}, \ldots, a_{r} ; F}(D)$. Then, for every $\varepsilon>0$ and $h>0$,

$$
\liminf _{N \rightarrow \infty} \frac{1}{M+1} \#\left\{N \leqslant k \leqslant N+M: \sup _{s \in K}\left|F\left(\zeta\left(s+i h \gamma_{k}\right)\right)-f(s)\right|<\varepsilon\right\}>0 .
$$

Moreover "lim inf" can be replaced by "lim" for all but at most countably many $\varepsilon>0$.

For example, the operators $F(g)=\sin g$ and $F(g)=\sinh g$ satisfy the hypotheses of Theorem 3 with $a_{1}=-1$ and $a_{2}=1$.

The proofs of Theorems 2 and 3 use probabilistic limit theorems for measures in the space $H(D)$. Denote by $\mathcal{B}(\mathbb{X})$ the Borel $\sigma$-field of the space $\mathbb{X}$. The main limit theorem will be proved for

$$
P_{N, M, h}(A)=\frac{1}{M+1} \#\left\{N \leqslant k \leqslant N+M: \zeta\left(s+i h \gamma_{k}\right) \in A\right\}, A \in \mathcal{B}(H(D)),
$$

as $N \rightarrow \infty$. We divide its proof into four sections.

\section{A Limit Theorem on the Torus}

Denote by $\gamma$ the unit circle on the complex plane, by $\mathbb{P}$ the set of all prime numbers, and define the set

$$
\Omega=\prod_{p \in \mathbb{P}} \gamma_{p}
$$

where $\gamma_{p}=\gamma$ for all $p \in \mathbb{P}$. With the product topology and pointwise multiplication, the torus $\Omega$ is a compact topological Abelian group. Therefore, on $(\Omega, \mathcal{B}(\Omega))$, the probability Haar measure $m_{H}$ can be defined, and we have the probability space $\left(\Omega, \mathcal{B}(\Omega), m_{H}\right)$. Denote by $\omega(p)$ the $p$ th component of an element $\omega \in \Omega, p \in \mathbb{P}$.

In this section, we will prove a limit theorem for

$$
Q_{N, M, h}(A)=\frac{1}{M+1} \#\left\{N \leqslant k \leqslant N+M:\left(p^{-i h \gamma_{k}}: p \in \mathbb{P}\right) \in A\right\}, A \in \mathcal{B}(\Omega),
$$

as $N \rightarrow \infty$.

Before the statement of a limit theorem for $Q_{N, M, h}$ as $N \rightarrow \infty$, we will recall some useful results that will be used in its proof. Denote by $N(T)$ the number of non-trivial zeros of $\zeta(s)$ in the region $\{s \in \mathbb{C}: 0<t<T\}$.

Lemma 1 (von Mongoldt formula). For $T \rightarrow \infty$,

$$
N(T)=\frac{T}{2 \pi} \log \frac{T}{2 \pi \mathrm{e}}+O(\log T) .
$$

For the proof, see, for example, [17].

Denote by $N(\sigma, T)$ the number of zeros $\rho=\beta+i \gamma$ of $\zeta(s)$ with $\beta>\sigma$ and $|\gamma|<T$. 
Lemma 2. Suppose that $H \geqslant T^{\alpha}$ with $\alpha>27 / 82$. Then, for $1 / 2<\sigma<1$, uniformly in $\sigma$,

$$
N(\sigma, T+H)-N(\sigma, T)=O\left(\frac{H}{\sigma-1 / 2}\right) .
$$

Proof of the lemma can be found in [18].

For positive $u \neq 1$, denote by $\Lambda(u)$ the von Mongoldt function if $u \in \mathbb{N} \backslash\{1\}$, and zero, otherwise.

Lemma 3. For positive $x \neq 1$ and $T \rightarrow \infty$,

$$
\sum_{0<\gamma_{k}<T} x^{\rho_{k}}=\left(\Lambda(x)-x \Lambda\left(\frac{1}{x}\right)\right) \frac{T}{2 \pi}+O\left(T^{(1 / 2)+\varepsilon}\right)
$$

with every $\varepsilon>0$.

Proof. The lemma is Theorem 2 of [19] with $a=0$.

Lemma 4. Suppose that $N^{1 / 2+\varepsilon} \leqslant M \leqslant N$ with $\varepsilon>0$. Then, for positive $x \neq 1$, as $N \rightarrow \infty$,

$$
\sum_{k=N}^{N+M} x^{\rho_{k}} \ll x \frac{M}{\sqrt{\log M}}
$$

Proof. Since

$$
\frac{N}{\log N} \ll \gamma_{N} \ll \frac{N}{\log N}
$$

in view of Lemma 3,

$$
\sum_{\gamma_{N}<\gamma \leqslant \gamma_{N+M}} x^{\rho}=\left(\Lambda(x)-x \Lambda\left(\frac{1}{x}\right)\right) \frac{\gamma_{N+M}-\gamma_{N}}{2 \pi}+O\left(\frac{N^{1 / 2+\varepsilon}}{\sqrt{\log N}}\right) .
$$

An application of Lemma 1 gives

$$
N+M=\sum_{\gamma \leqslant \gamma_{N+M}} 1=\frac{\gamma_{N+M}}{2 \pi} \log \frac{\gamma_{N+M}}{2 \pi \mathrm{e}}+O(\log N)
$$

and

$$
N=\sum_{\gamma \leqslant \gamma_{N}} 1=\frac{\gamma_{N}}{2 \pi} \log \frac{\gamma_{N}}{2 \pi \mathrm{e}}+O(\log N)
$$

Therefore,

$$
\gamma_{N+M}=\frac{2 \pi(N+M)}{\log \left(\gamma_{N+M} /(2 \pi \mathrm{e})\right)}+O(1)
$$

and

$$
\gamma_{N}=\frac{2 \pi N}{\log \left(\gamma_{N} /(2 \pi \mathrm{e})\right)}+O(1)
$$

Hence,

$$
\gamma_{N+M}-\gamma_{N} \leqslant \frac{2 \pi(N+M)}{\log \left(\gamma_{N} /(2 \pi \mathrm{e})\right)}-\frac{2 \pi N}{\log \left(\gamma_{N} /(2 \pi \mathrm{e})\right)}+O(1) \ll \frac{M}{\log N}+O(1) \ll \frac{M}{\log M} .
$$

This together with Equation (3) proves the lemma.

Now, we state the limit theorem for $Q_{N, M, h}$. 
Theorem 4. Suppose that, for any $\varepsilon>0, N^{1 / 2+\varepsilon} \leqslant M \leqslant N$. Then $Q_{N, M, h}$ converges weakly to the Haar measure $m_{H}$ as $N \rightarrow \infty$.

Proof. Denote by $g_{N, M, h}(\underline{k}), \underline{k}=\left(k_{p}: k_{p} \in \mathbb{Z}, p \in \mathbb{P}\right)$, the Fourier transform of $Q_{N, M, h}$, i.e.,

$$
g_{N, M, h}(\underline{k})=\int_{\Omega}\left(\prod_{p \in \mathbb{P}}^{*} \omega^{k_{p}}(p)\right) \mathrm{d} Q_{N, M, h}
$$

where the star " $*$ " means that only a finite number of integers $k_{p}$ are distinct from zero. Thus, by the definition of $Q_{N, M, h}$

$$
g_{N, M, h}(\underline{k})=\frac{1}{M+1} \sum_{k=N}^{N+M} \exp \left\{-i h \gamma_{k} \sum_{p \in \mathbb{P}}^{*} k_{p} \log p\right\}
$$

Clearly,

$$
g_{N, M, h}(\underline{0})=1 \text {. }
$$

Now, suppose that $k \neq \underline{0}$. Since the set $\{\log p: p \in \mathbb{P}\}$ is linearly independent within the field of rational numbers $\mathbb{Q}$, in that case we have

$$
a \stackrel{\text { def }}{=} \sum_{p \in \mathbb{P}}^{*} k_{p} \log p \neq 0
$$

Thus, we will estimate the sum

$$
\sum_{k=N}^{N+M} \exp \left\{i h a \gamma_{k}\right\}
$$

It is easily seen that

$$
\begin{aligned}
\sum_{k=N}^{N+M}\left(\exp \left\{h a \beta_{k}\right\}-\exp \left\{\frac{1}{2} h a\right\}\right) & \ll_{h, a} \sum_{k=N}^{N+M}\left|\exp \left\{h a\left(\beta_{k}-\frac{1}{2}\right)\right\}-1\right| \\
& \ll_{h, a} \sum_{k=N}^{N+M}\left|\beta_{k}-\frac{1}{2}\right|=\sum_{k=N}^{N+M}\left|\beta_{k}-\frac{1}{2}\right|+\sum_{k=N}^{N+M}\left|\beta_{k}-\frac{1}{2}\right|
\end{aligned}
$$

where $\left|\beta_{k}-1 / 2\right| \leqslant 1 / \log \log M$ in $\sum^{\prime}$, and $\left|\beta_{k}-1 / 2\right|>1 / \log \log M$ in $\sum^{\prime \prime}$. Obviously,

$$
\sum_{k=N}^{N+M}\left|\beta_{k}-\frac{1}{2}\right| \leqslant \frac{M}{\log \log M}
$$

Therefore, by Lemma 2 and estimate (2),

$$
\sum_{k=N}^{N+M}\left|\beta_{k}-\frac{1}{2}\right| \ll \sum_{\gamma_{N}<\gamma \leqslant \gamma_{N+M}}^{\prime \prime} 1 \ll \frac{M \log \log M}{\log M} .
$$

This, and estimates (7) and (8) show that

$$
\sum_{k=N}^{N+M} \exp \left\{\left(\beta_{k}+i \gamma_{k}\right) h a\right\}-\sum_{k=N}^{N+M} \exp \left\{\left(\frac{1}{2}+i \gamma_{k}\right) h a\right\} \ll_{h, a} \frac{M}{\log \log M}
$$

Lemma 4 with $x=\exp \{h a\}$ implies

$$
\sum_{k=N}^{N+M} \exp \left\{\left(\beta_{k}+i \gamma_{k}\right) h a\right\} \ll_{h, a} \frac{M}{\sqrt{\log M}}
$$


Therefore, in view of estimate (9),

$$
\sum_{k=N}^{N+M} \exp \left\{i h a \gamma_{k}\right\} \ll_{h, a} \sum_{k=N}^{N+M} \exp \left\{\left(\frac{1}{2}+i \gamma_{k}\right) h a\right\} \ll_{h, a} \frac{M}{\log \log M} .
$$

Thus, by Equation (5),

$$
g_{N, M, h}(\underline{k}) \ll_{h, a} \frac{1}{\log \log M} .
$$

This together with Equation (6) shows that

$$
\lim _{N \rightarrow \infty} g_{N, M, h}(\underline{k})= \begin{cases}1 & \text { if } \underline{k}=\underline{0}, \\ 0 & \text { if } \underline{k} \neq \underline{0},\end{cases}
$$

and the lemma is proved because the right-hand side of the latter equality is the Fourier transform of the measure $m_{H}$.

\section{A Limit Theorem for Absolutely Convergent Series}

Let $\theta>1 / 2$ be a fixed number, and $v_{n}(m)=\exp \left\{-(m / n)^{\theta}\right\}$ for $m, n \in \mathbb{N}$. Extend the function $\omega(p)$ to the set $\mathbb{N}$ by setting

$$
\omega(m)=\prod_{\substack{p^{l} \mid m \\ p^{l+1} \nmid m}} \omega^{l}(p),
$$

and define

$$
\zeta_{n}(s)=\sum_{m=1}^{\infty} \frac{v_{n}(m)}{m^{s}}
$$

and

$$
\zeta_{n}(s, \omega)=\sum_{m=1}^{\infty} \frac{\omega(m) v_{n}(m)}{m^{s}} .
$$

Then the latter series are absolutely convergent for $\sigma>1 / 2$ [5]. Consider the function $u_{n}: \Omega \rightarrow$ $H(D)$ defined by

$$
u_{n}(\omega)=\zeta_{n}(s, \omega) .
$$

The absolute convergence of the series $\zeta_{n}(s, \omega)$ implies the continuity of $u_{n}$.

For $A \in \mathcal{B}(H(D))$, define

$$
P_{N, M, n, h}(A)=\frac{1}{M+1} \#\left\{N \leqslant k \leqslant N+M: \zeta_{n}\left(s+i h \gamma_{k}\right) \in A\right\} .
$$

Theorem 5. Suppose that $N^{1 / 2+\varepsilon} \leqslant M \leqslant N$. Then $P_{N, M, n, h}$ converges weakly to the measure $m_{H} u_{n}^{-1} \stackrel{\text { def }}{=} V_{n}$.

Proof. The theorem follows from the equality

$$
P_{N, M, n, h}(A)=Q_{N, M, h}\left(u_{n}^{-1} A\right)=Q_{N, M, h} u_{n}^{-1}(A), \quad A \in \mathcal{B}(H(D)),
$$

continuity of the function $u_{n}$, Theorem 4 and Theorem 5.1 of [20].

The weak convergence of $P_{N, M, h}$ is closely connected to that of $V_{n}$ as $n \rightarrow \infty$. Define

$$
\zeta(s, \omega)=\prod_{p \in \mathbb{P}}\left(1-\frac{\omega(p)}{p^{s}}\right)^{-1} .
$$


Then $\zeta(s, \omega)$ is an $H(D)$-valued random element on the probability space $\left(\Omega, \mathcal{B}(\Omega), m_{H}\right)$ [5]. We recall that the latter infinite product, for almost all $\omega$, is uniformly convergent on compact subsets $K \subset D$. Denote by $P_{\zeta}$ the distribution of the random element $\zeta(s, \omega)$, i.e.,

$$
P_{\zeta}(A)=m_{H}\{\omega \in \Omega: \zeta(s, \omega) \in A\}, \quad A \in \mathcal{B}(H(D)) .
$$

The following statement is very important.

Proposition 1. The probability measure $V_{n}$ converges weakly to measure $P_{\zeta}$ as $n \rightarrow \infty$.

Proof. For $A \in \mathcal{B}(H(D))$, define

$$
R_{T}(A)=\frac{1}{T} \operatorname{meas}\{\tau \in[0, T]: \zeta(s+i \tau) \in A\} .
$$

It is known that $R_{T}$, as $T \rightarrow \infty$, converges weakly to $P_{\zeta}$ [5]. Moreover, $R_{T}$, as $T \rightarrow \infty$, and $V_{n}$, as $n \rightarrow \infty$, converge weakly to the same probability measure on $(H(D), \mathcal{B}(H(D)))$. Thus, $V_{n}$ converges weakly to $P_{\zeta}$ as $n \rightarrow \infty$.

\section{Mean Square Estimates in Short Intervals}

To derive the weak convergence of $P_{N, M, h}$ from that of $P_{N, M, n, h}$ as $N \rightarrow \infty$, the estimate for

$$
\sum_{k=N}^{N+M}\left|\zeta\left(\sigma+i h \gamma_{k}+i t\right)\right|^{2}
$$

with $t \in \mathbb{R}$ is needed.

We will use the following mean square estimate in short intervals.

Lemma 5. Suppose that $\sigma, 1 / 2<\sigma<1$, is fixed and $T^{1 / 3}(\log T)^{26 / 15} \leqslant H \leqslant T$. Then, uniformly in $H$,

$$
\int_{T-H}^{T+H}|\zeta(\sigma+i t)|^{2} \ll_{\sigma} H
$$

The lemma follows from Theorem 7.1 of [21], and was used in [16].

Lemma 6. Suppose that $N^{1 / 2+\varepsilon} \leqslant M \leqslant N$ and estimate (2) is true. Then, for every fixed $\sigma, 1 / 2<\sigma<1$, $h>0$ and $t \in \mathbb{R}$,

$$
\sum_{k=N}^{N+M}\left|\zeta\left(\sigma+i h \gamma_{k}+i t\right)\right| \ll_{\sigma, h} M(1+|t|)
$$

Proof. We will apply the Gallagher lemma connecting discrete mean squares with those continuous of some functions; for the proof, see Lemma 1.4 of [22]. Let $T_{0}, T \geqslant \delta>0$ be real numbers, $\mathcal{T} \neq \varnothing$ be a finite set in the interval $\left[T_{0}+\delta / 2, T_{0}+T-\delta / 2\right]$,

$$
N_{\delta}(x)=\sum_{\substack{t \in \mathcal{T} \\|t-x|<\delta}} 1
$$

and let $S(x)$ be a complex-valued continuous function on $\left[T_{0}, T+T_{0}\right]$ having a continuous derivative on $\left(T_{0}, T+T_{0}\right)$. Then the Gallagher lemma asserts that

$$
\sum_{t \in \mathcal{T}} N_{\delta}^{-1}(t)|S(t)|^{2} \leqslant \frac{1}{\delta} \int_{T_{0}}^{T_{0}+T}|S(x)|^{2} \mathrm{~d} x+\left(\int_{T_{0}}^{T_{0}+T}|S(x)|^{2} \mathrm{~d} x \int_{T_{0}}^{T_{0}+T}\left|S^{\prime}(x)\right|^{2} \mathrm{~d} x\right)^{1 / 2} .
$$


We apply the Gallagher lemma for the function $\zeta\left(s+i k h \gamma_{k}+i t\right)$. In our case $\delta=c / \log N$, $T_{0}=h \gamma_{N}-\delta / 2, T=h \gamma_{N+M}-h \gamma_{N}+\delta / 2$ and $\mathcal{T}=\left\{h \gamma_{N}, h \gamma_{N+1}, \ldots, h \gamma_{N+M}\right\}$. By estimate (2), we have

$$
\sum_{k=N}^{N+M} N_{\delta}\left(h \gamma_{k}\right)=\sum_{\substack{k=N \\\left|\gamma_{k}-\gamma_{l}\right|<c /(h \log N)}}^{N+M} \sum_{l=N}^{N+M} 1 \ll_{h} M .
$$

Now, an application of the Gallagher lemma gives

$$
\begin{aligned}
\sum_{k=N}^{N+M}\left|\zeta\left(\sigma+i h \gamma_{k}+i t\right)\right| & =\sum_{k=N}^{N+M} \sqrt{N_{\delta}\left(h \gamma_{k}\right) N^{-1}\left(h \gamma_{k}\right)}\left|\zeta\left(\sigma+i h \gamma_{k}+i t\right)\right| \\
\leqslant & \left(\sum_{k=N}^{N+M} N_{\delta}\left(h \gamma_{k}\right) \sum_{k=N}^{N+M} N^{-1}\left(h \gamma_{k}\right)\left|\zeta\left(\sigma+i h \gamma_{k}+i t\right)\right|^{2}\right)^{1 / 2} \\
& \ll h \sqrt{M} \sqrt{\log N}\left(\int_{h \gamma_{N}-\delta / 2}^{h \gamma_{N+M}}|\zeta(\sigma+i \tau+i t)|^{2} \mathrm{~d} \tau\right. \\
& \left.+\left(\int_{h \gamma_{N}-\delta}^{h \gamma_{N+M}}|\zeta(\sigma+i \tau+i t)|^{2} \mathrm{~d} \tau \int_{h \gamma_{N}-\delta}^{h \gamma_{N+M}}\left|\zeta^{\prime}(\sigma+i \tau+i t)\right|^{2} \mathrm{~d} \tau\right)^{1 / 2}\right)^{1 / 2} .
\end{aligned}
$$

The estimate (4) gives with certain $c_{h}>0$

$$
\int_{h \gamma_{N}-\delta}^{h \gamma_{N+M}}|\zeta(\sigma+i \tau+i t)|^{2} \mathrm{~d} t \ll \int_{h \gamma_{N}-\delta-|t|}^{h \gamma_{N}+c_{h}(M / \log M)+|t|}|\zeta(\sigma+i \tau)|^{2} \mathrm{~d} \tau .
$$

If $c_{h}(M / \log M)+|t| \leqslant h \gamma_{N}$, then, in view of Lemma 5, the right-hand side of (13) is

$$
\ll_{\sigma, h} \frac{M}{\log M}+|t| \ll_{\sigma, h} \frac{M}{\log M}(1+|t|) .
$$

If $c_{h}(M / \log M)+|t|>h \gamma_{N}$, then

$$
h \gamma_{N}+c_{h} \frac{M}{\log M}+|t|<2\left(c_{h} \frac{M}{\log M}+|t|\right)
$$

and

$$
h \gamma_{N}-\delta>h \gamma_{N}-2 c_{h} \frac{M}{\log M}-2|t|>-h \gamma_{N} c_{h} \frac{M}{\log M}-|t|>-2\left(c_{h} \frac{M}{\log M}+|t|\right) .
$$

Thus, in this case,

$$
\int_{h \gamma_{N}-\delta}^{h \gamma_{N+M}}|\zeta(\sigma+i \tau+i t)|^{2} \mathrm{~d} \tau \ll_{h} \int_{-2\left(c_{h}(M / \log M)+|t|\right)}^{2\left(c_{h}(M / \log M)+|t|\right)}|\zeta(\sigma+i \tau)|^{2} \mathrm{~d} \tau \ll_{\sigma, h} \frac{M}{\log M}(1+|t|) .
$$

This together with estimate (13) shows that

$$
\int_{h \gamma_{N}-\delta}^{h \gamma_{N+M}}|\zeta(\sigma+i \tau+i t)|^{2} \mathrm{~d} \tau \ll_{\sigma, h} \frac{M}{\log M}(1+|t|) .
$$

Estimate (14) and an application of the Cauchy integral formula lead to the bound

$$
\int_{h \gamma_{N}-\delta}^{h \gamma_{N+M}}\left|\zeta^{\prime}(\sigma+i \tau+i t)\right|^{2} \mathrm{~d} \tau \ll_{\sigma, h} \frac{M}{\log M}(1+|t|)
$$

This, estimate (14) and (12) prove the lemma.

Now, we are ready to state an approximation lemma. 


\section{Approximation in the Mean}

Denote by $\rho$ the metric in $H(D)$ which induces the topology of uniform convergence on compacta. More precisely, for $g_{1}, g_{2} \in H(D)$,

$$
\rho\left(g_{1}, g_{2}\right)=\sum_{l=1}^{\infty} 2^{-l} \frac{\sup _{s \in K_{l}}\left|g_{1}(s)-g_{2}(s)\right|}{1+\sup _{s \in K_{l}}\left|g_{1}(s)-g_{2}(s)\right|},
$$

where $\left\{K_{l}: l \in \mathbb{N}\right\} \subset D$ is a sequence of compact subsets such that

$$
D=\bigcup_{l=1}^{\infty} K_{l},
$$

$K_{l} \subset K_{l+1}$ for all $l \in \mathbb{N}$, and every compact $K \subset D$ lies in a certain $K_{l}$.

Lemma 7. Suppose that $N^{1 / 2+\varepsilon} \leqslant M \leqslant N$ and (2) is true. Then, for every $h>0$,

$$
\lim _{n \rightarrow \infty} \limsup _{N \rightarrow \infty} \frac{1}{M+1} \sum_{k=N}^{N+M} \rho\left(\zeta\left(s+i h \gamma_{k}\right), \zeta_{n}\left(s+i h \gamma_{k}\right)\right)=0
$$

Proof. In view of the definition of the metric $\rho$, it suffices to show that, for every compact $K \subset D$,

$$
\lim _{n \rightarrow \infty} \limsup _{N \rightarrow \infty} \frac{1}{M+1} \sum_{k=N}^{N+M} \sup _{s \in K}\left|\zeta\left(s+i h \gamma_{k}\right)-\zeta_{n}\left(s+i h \gamma_{k}\right)\right|=0 .
$$

Thus, let $K \subset D$ be a fixed compact set. Denote the points of $K$ by $s=\sigma+i v$, and fix $\varepsilon>0$ such that $\mathbf{1} / \mathbf{2}+\mathbf{2} \varepsilon \leqslant \sigma \leqslant \mathbf{1}-\varepsilon$ for $s \in K$. It is known [5] that

$$
\zeta_{n}(s)=\frac{1}{2 \pi i} \int_{\theta-i \infty}^{\theta+i \infty} \zeta(s+z) l_{n}(z) \frac{\mathrm{d} z}{z},
$$

where

$$
l_{n}(s)=\frac{s}{\theta} \Gamma(s / \theta) n^{s},
$$

$\Gamma(s)$ is the Euler gamma-function, and $\theta$ comes from the definition of $v_{n}(m)$. Let $\theta_{1}>0$. From this, we have

$$
\zeta(s)-\zeta_{n}(s)=\frac{1}{2 \pi i} \int_{-\theta-i \infty}^{-\theta+i \infty} \zeta(s+z) l_{n}(z) \frac{\mathrm{d} z}{z}+R_{n}(s),
$$

with

$$
R_{n}(s)=\frac{l_{n}(1-s)}{1-s} .
$$

Therefore, as in the proof of Lemma 12 of [16], we find that

$$
\begin{aligned}
& \frac{1}{M+1} \sum_{k=N}^{N+M} \sup _{s \in K}\left|\zeta\left(s+i h \gamma_{k}\right)-\zeta_{n}\left(s+i h \gamma_{k}\right)\right| \\
& \quad \ll \int_{-\infty}^{\infty} \frac{1}{M} \sum_{k=N}^{N+M}\left|\zeta\left(\frac{1}{2}+\varepsilon+i\left(h \gamma_{k}+t\right)\right)\right| \sup _{s \in K} \frac{\left|l_{n}(1 / 2+\varepsilon-s+i t)\right|}{|1 / 2+\varepsilon-s+i t|} \mathrm{d} t \\
& \quad+\frac{1}{M} \sum_{k=N}^{N+M} \sup _{s \in K}\left|R_{n}\left(s+i h \gamma_{k}\right)\right| \stackrel{\text { def }}{=} I_{1}+I_{2} .
\end{aligned}
$$

Denote by $c_{1}, c_{2}, \ldots$ positive constants. In view of the well-known estimate

$$
\Gamma(\sigma+i t) \ll \exp \left\{-c_{1}|t|\right\},
$$


we find that

$$
\frac{\left|l_{n}(1 / 2+\varepsilon-s+i t)\right|}{|1 / 2+\varepsilon-s+i t|} \ll n^{-\varepsilon} \exp \left\{-c_{2}|t-v|\right\} \ll_{K, \varepsilon} n^{-\varepsilon} \exp \left\{-c_{3}|t|\right\} .
$$

Therefore, by Lemma 5,

$$
I_{1} \ll_{K, \varepsilon} n^{-\varepsilon} \int_{-\infty}^{\infty}(1+|t|) \exp \left\{-c_{3}|t|\right\} \mathrm{d} t \ll_{K, \varepsilon} n^{-\varepsilon} .
$$

Similarly, taking into account inequality (17), we find

$$
\begin{aligned}
I_{2} & \ll \frac{n^{1 / 2-2 \varepsilon}}{M} \sum_{k=N}^{N+M} \exp \left\{-c_{4}\left|h \gamma_{k}-v\right|\right\} \ll_{K} \frac{n^{1 / 2-2 \varepsilon}}{M} \sum_{k=N}^{N+M} \exp \left\{-c_{5} h \gamma_{k}\right\} \\
& \ll_{K} \frac{n^{1 / 2-2 \varepsilon}}{M} \sum_{k=N}^{N+M} \exp \left\{-c_{6} h(k / \log k)\right\} \ll_{K, h} \frac{n^{1 / 2-2 \varepsilon}}{M} .
\end{aligned}
$$

This, Equations (18) and (16) prove (15).

\section{A Limit Theorem for $\zeta(s)$}

Using the results of Sections 3 and 4 leads to a limit theorem for $P_{N, M, h}$.

Theorem 6. Suppose that $N^{1 / 2+\varepsilon} \leqslant M \leqslant N$ and estimate (2) is true. Then $P_{N, M, h}$ converges weakly to $P_{\zeta}$ as $N \rightarrow \infty$.

Proof. In a certain probability space with measure $\mu$ define the random variable $\theta_{N, M, h}$ with the distribution

$$
\mu\left\{\theta_{N, M, h}=h \gamma_{k}\right\}=\frac{1}{M+1}, \quad k=N, N+1, \ldots, N+M,
$$

and consider the $H(D)$-valued random element

$$
X_{N, M, n, h}=X_{N, M, n, h}(s)=\zeta_{n}\left(s+i \theta_{N, M, h}\right) .
$$

Moreover, let $X_{n}=X_{n}(s)$ be the $H(D)$-valued random element with the distribution $V_{n}$. Then, by Theorem 5,

$$
X_{N, M, n, h} \underset{N \rightarrow \infty}{\stackrel{\mathcal{D}}{\longrightarrow}} X_{n}
$$

where $\stackrel{\mathcal{D}}{\rightarrow}$ denotes the convergence in distribution. Moreover, by Proposition 1 ,

$$
X_{n} \underset{n \rightarrow \infty}{\stackrel{\mathcal{D}}{\longrightarrow}} P_{\zeta}
$$

Define one more $H(D)$-valued random element

$$
X_{N, M, h}=X_{N, M, h}(s)=\zeta\left(s+i \theta_{N, M, h}\right) .
$$

Then, using Lemma 7, we find that, for every $\varepsilon>0$,

$$
\begin{aligned}
\lim _{n \rightarrow \infty} & \limsup _{N \rightarrow \infty} \mu\left\{\rho\left(X_{N, M, h}, X_{N, M, n, h}\right) \geqslant \varepsilon\right\} \\
& \leqslant \lim _{n \rightarrow \infty} \limsup _{N \rightarrow \infty} \frac{1}{\varepsilon(M+1)} \sum_{k=N}^{N+M} \rho\left(\zeta\left(s+i h \gamma_{k}\right), \zeta_{n}\left(s+i h \gamma_{k}\right)\right)=0 .
\end{aligned}
$$


Now, this, Equations (19) and (20) together with Theorem 4.2 of [20] show that

$$
X_{N, M, h} \underset{n \rightarrow \infty}{\stackrel{\mathcal{D}}{\longrightarrow}} P_{\zeta}
$$

and theorem is proved.

For $A \in \mathcal{B}(H(D))$, define

$$
P_{N, M, h, F}(A)=\frac{1}{M+1} \#\left\{N \leqslant k \leqslant N+M: F\left(\zeta\left(s+i h \gamma_{k}\right)\right) \in A\right\} .
$$

Corollary 1. Suppose that $F: H(D) \rightarrow H(D)$ is a continuous operator, and (2) is true. Then $P_{N, M, h, F}$ converges weakly to $P_{\zeta} F^{-1}$ as $N \rightarrow \infty$.

Proof. The corollary follows from Theorem 5 , continuity of $F$, equality

$$
P_{N, M, h, F}=P_{N, M, h} F^{-1}
$$

and Theorem 5.1 of [20].

\section{Proof of Universality}

Theorems 2 and 3 are derived from Theorem 6 and Corollary 1, respectively, by using the Mergelyan theorem on the approximation of analytic functions by polynomials [23].

Proof of Theorem 2. We recall that

$$
S=\{g \in H(D): \text { either } g(s) \neq 0 \text { for all } s \in D, \text { or } g(s) \equiv 0\},
$$

It is well known, see, for example, [5], that the support of the measure $P_{\zeta}$ is the set $S$. Define the set

$$
G_{\varepsilon}=\left\{g \in H(D): \sup _{s \in K}\left|g(s)-\mathrm{e}^{p(s)}\right|<\frac{\varepsilon}{2}\right\},
$$

where $p(s)$ is a polynomial. Obviously, $\mathrm{e}^{p(s)} \in S$. Therefore, $G_{\varepsilon}$ is an open neighbourhood of an element of the support of the measure $P_{\zeta}$. Thus, by a property of the support,

$$
P_{\zeta}\left(G_{\varepsilon}\right)>0 .
$$

This, Theorem 6 and the equivalent of weak convergence in terms of open sets show that

$$
\liminf _{N \rightarrow \infty} P_{N, M, h}\left(G_{\varepsilon}\right) \geqslant P_{\zeta}\left(G_{\varepsilon}\right)>0 .
$$

Hence, by the definition of $P_{N, M, h}$ and $G_{\varepsilon}$,

$$
\liminf _{N \rightarrow \infty} \frac{1}{M+1} \#\left\{N \leqslant k \leqslant N+M: \sup _{s \in K}\left|\zeta\left(s+i h \gamma_{k}\right)-\mathrm{e}^{p(s)}\right|<\frac{\varepsilon}{2}\right\}>0 .
$$

Now, we apply the Mergelyan theorem and choose the polynomial $p(s)$ satisfying

$$
\sup _{s \in K}\left|f(s)-\mathrm{e}^{p(s)}\right|<\frac{\varepsilon}{2} .
$$

This and inequality (22) prove the first part of the theorem. 
To prove the second part of the theorem, define the set

$$
\hat{G}_{\varepsilon}=\left\{g \in H(D): \sup _{s \in K}|g(s)-f(s)|<\varepsilon\right\} .
$$

Then the set $\hat{G}_{\varepsilon}$ is a continuity set of the measure $P_{\zeta}$ for all but at most countably many $\varepsilon>0$. This remark, Theorem 6 and the equivalent of weak convergence of probability measures in terms of open sets show that

$$
\lim _{N \rightarrow \infty} P_{N, M, h}\left(\hat{G}_{\varepsilon}\right)=P_{\zeta}\left(\hat{G}_{\varepsilon}\right)
$$

for all but at most countably many $\varepsilon>0$. Inequality (23) implies the inclusion $G_{\varepsilon} \subset \hat{G}_{\varepsilon}$. Therefore, in view of inequality (21), we have $P_{\zeta}\left(\hat{G}_{\varepsilon}\right)>0$. This, Equation (24) and the definitions of $P_{N, M, h}$ and $\hat{G}_{\varepsilon}$ prove the second part of the theorem.

Proof of Theorem 3. Denote by $S_{F}$ the support of the measure $P_{\zeta} F^{-1}$. We observe that $S_{F}$ contains the closure of the set $H_{a_{1}, \ldots, a_{r} ; F}(D)$. Actually, let $g \in H_{a_{1}, \ldots, a_{r} ; F}(D)$ and $G$ be any open neighborhood of $g$. Then the set $F^{-1} G$ is open as well, and lies in $S$. Hence, $P_{\zeta}\left(F^{-1} G\right)>0$ because $S$ is the support of $P_{\zeta}$. Therefore,

$$
P_{\zeta} F^{-1}(G)=P_{\zeta}\left(F^{-1} G\right)>0 .
$$

This shows that $S_{F}$ contains the set $H_{a_{1}, \ldots, a_{r} ; F}(D)$ and its closure.

Case $r=1$. By the Mergelyan theorem, there exists a polynomial $p(s)$ such that

$$
\sup _{s \in K}|f(s)-p(s)|<\frac{\varepsilon}{2} .
$$

Then, $p(s) \neq a_{1}$ for all $s \in K$ if $\varepsilon$ is small enough. Therefore, by the Mergelyan theorem again, we find a polynomial $q(s)$ such that

$$
\sup _{s \in K}\left|\left(p(s)-a_{1}\right)-\mathrm{e}^{q(s)}\right|<\frac{\varepsilon}{4} .
$$

Since $g_{1}(s) \stackrel{\text { def }}{=} \mathrm{e}^{q(s)}+a_{1} \in H_{a_{1} ; F}(D)$, the set

$$
\mathcal{G}_{\varepsilon}=\left\{g \in H(D): \sup _{s \in K}\left|g(s)-g_{1}(s)\right|<\frac{\varepsilon}{2}\right\}
$$

is an open subset of $S_{F}$. Hence,

$$
P_{\zeta} F^{-1}\left(\mathcal{G}_{\varepsilon}\right)>0 .
$$

This inequality together with Corollary 1 , inequalities (25) and (26) prove the theorem in the case of the lower density.

In the case of density, consider the set $\hat{G}_{\varepsilon}$ defined in the proof of Theorem 2 which is a continuity set of the measure $P_{\zeta} F^{-1}$ for all but at most countably many $\varepsilon>0$. Therefore, by Corollary 1 ,

$$
\lim _{N \rightarrow \infty} P_{N, M, h, F}\left(\hat{G}_{\varepsilon}\right)=P_{\zeta} F^{-1}\left(\hat{G}_{\varepsilon}\right) .
$$

Inequalities (25) and (26) show that $\mathcal{G}_{\varepsilon} \subset \hat{G}_{\varepsilon}$. Thus, by inequality (27), $P_{\zeta} F^{-1}\left(\hat{G}_{\varepsilon}\right)>0$. This, Equation (28) and the definitions of $P_{N, M, h, F}$ and $\hat{G}_{\varepsilon}$ prove the theorem in the case of density.

Case $r \geqslant 2$. In this case, the function $f(s)$ lies in $S_{F}$. Therefore, the Mergelyan theorem is not needed, and the theorem follows immediately from Corollary 1. 
Author Contributions: Conceptualization, A.L. and D.Š.; methodology, A.L. and D.Š.; investigation, A.L. and D.Š.; writing—original draft preparation, A.L. and D.Š.; writing-review and editing, A.L. and D.Š. All authors have read and agreed to the published version of the manuscript.

Funding: The research of the first author is funded by the European Social Fund (project number 09.3.3-LMT-K-712-01-0037) under grant agreement with the Research Council of Lithuania (LMT LT).

Conflicts of Interest: The authors declare no conflict of interest.

\section{References}

1. Bohr, H.; Courant, R. Neue Anwendungen der Theorie der Diophantischen Approximationen auf die Riemannsche Zetafunktion. J. Reine Angew. Math. 1914, 144, 249-274.

2. Voronin, S.M. Theorem on the "universality" of the Riemann zeta-function. Izv. Ross. Akad. Nauk. 1975, 9, 443-453. [CrossRef]

3. Bagchi, B. The Statistical Behaviour and Universality Properties of the Riemann Zeta-Function and Other Allied Dirichlet Series. Ph.D. Thesis, Indian Statistical Institute, Calcutta, India, 1981.

4. Gonek, S.M. Analytic Properties of Zeta and L-Functions. Ph.D. Thesis, University of Michigan, Ann Arbor, MI, USA, 1975.

5. Laurinčikas, A. Limit Theorems for the Riemann Zeta-Function; Kluwer Academic Publishers: Dordrecht, The Netherlands; Boston, MA, USA; London, UK, 1996.

6. Steuding, J. Value-Distribution of L-Functions; Lecture Notes Math; Springer: Berlin/Heidelberg, Germany; New York, NY, USA, 2007; Volume 1877.

7. Reich, A. Werteverteilung von Zetafunktionen. Arch. Math. 1980, 34, 440-451. [CrossRef]

8. Laurinčikas, A. Discrete universality of the Riemann zeta-function and uniform distribution modulo 1. Petersb. Math. J. 2019, 30, 103-110. [CrossRef]

9. Macaitiene, R. The discrete universality of the Riemann zeta-function with respect to uniformly distributed shifts. Arch. Math. 2017, 108, 271-281. [CrossRef]

10. Pańkowski, Ł. Joint universality for dependent L-functions. Ramanujan J. 2018, 45, 181-195. [CrossRef]

11. Garunkštis, R.; Laurinčikas, A. The Riemann hypothesis and universality of the Riemann zeta-function. Math. Slovaca 2018, 68, 741-748. [CrossRef]

12. Garunkštis, R.; Laurinčikas, A.; Macaitienè, R. Zeros of the Riemann zeta-function and its universality. Acta Arith. 2017, 181, 127-142. [CrossRef]

13. Montgomery, H.L. The pair correlation of zeros of the zeta-function. In Analytic Number Theory; Diamond, H.G., Ed.; American Mathematical Society: Providence, RI, USA, 1973; pp. 181-193.

14. Balčiūnas, A.; Garbaliauskienè, V.; Karaliūnaitè, J.; Macaitienè, R.; Petuškinaitè, J.; Rimkevičienè, A. Joint discrete approximation of a pair of analytic functions by periodic zeta-functions. Math. Modell. Anal. 2020, 25, 71-87. [CrossRef]

15. Laurinčikas, A.; Petuškinaitè, J. Universality of Dirichlet $L$-functions and non-trivial zeros of the Riemann zeta-function. Sb. Math. 2019, 210, 1753-1773. [CrossRef]

16. Laurinčikas, A. Universality of the Riemann zeta-function in short intervals. J. Number Theory 2019, 204, 279-295. [CrossRef]

17. Titchmarsh, E.C. The Theory of the Riemann zeta-Function, 2nd ed.; Heath-Brown, D.R., Ed.; Clarendon Press: Oxford, UK, 1986.

18. Karatsuba, A.A.; Voronin, S.M. The Riemann Zeta-Function; Walter de Gruiter: New York, NY, USA; Berlin, Germany, 1992.

19. Steuding, J. The roots of the equation $\zeta(s)=a$ are uniformly distributed modulo one. In Anal. Probab. Methods Number Theory; Laurinčikas, A., Ed.; TEV: Vilnius, Lithuania, 2012; pp. 243-249.

20. Billingsley, P. Convergence of Probability Measures; Willey: New York, NY, USA, 1968.

21. Ivič, A. The Riemann Zeta-Function: The Theory of the Riemann Zeta-Function with Applications; John Wiley \& Sons: New York, NY, USA, 1985. 
22. Montgomery, H.L. Topics in Multiplicative Number Theory; Lecture Notes in Mathematics; Springer: Berlin/Heidelberg, Germany; New York, NY, USA, 1971; Volume 227.

23. Mergelyan, S.N. Uniform Approximations to Functions of a Complex Variable; Series and Approximation; American Mathematical Society: Providence, RI, USA, 1969; pp. 294-391.

Publisher's Note: MDPI stays neutral with regard to jurisdictional claims in published maps and institutional affiliations.

(C) 2020 by the authors. Licensee MDPI, Basel, Switzerland. This article is an open access article distributed under the terms and conditions of the Creative Commons Attribution (CC BY) license (http://creativecommons.org/licenses/by/4.0/). 